www. revistadyo.com

\title{
Uso de videojuegos de simulación empresarial como complemento de aprendizaje en el área de Ingeniería de Organización
}

\author{
García-Miranda I , Duran Heras A
}

Recibido: 15 de Febrero de 2019

Aceptado: 27 de Mayo de 2019

https://doi.org/10.37610/dyo.v0i70.566

\section{Resumen}

Este artículo expone el uso de un videojuego de simulación empresarial como metodología activa de aprendizaje en alumnos de asignaturas del área de conocimiento de Ingeniería de Organización. Tras un proceso previo de análisis y valoración, se decide la aplicación de un videojuego de simulación empresarial para su aplicación en una asignatura cuyos conceptos son complejos. Se pretende que el uso del videojuego ayude a los alumnos a comprender mejor dichos conceptos además de mejorar la motivación. Entre todos los videojuegos analizados, se utiliza Virtonomics, que proporciona una serie de ventajas sobre el resto. Tras la implementación y evaluación de los resultados, se puede concluir que el uso de dicho videojuego cumple con los objetivos propuestos además de mejorar el espíritu emprendedor de los alumnos.

\section{Palabras clave}

Gamificación; Innovación docente; Educación superior; Enseñanza activa.

\section{Introducción}

En el año 1999 se inició el Proceso de Bolonia con el fin de armonizar en la Unión Europea los sistemas educativos de los distintos miembros. A pesar de no ser un proceso vinculante, la firma de dicho Proceso condujo a la creación del Espacio Europeo de Educación Superior (EEES). Entre los numerosos objetivos establecidos está el cambio en las metodologías docentes, con el fin de que el estudiante sea el centro del proceso de aprendizaje. Para ello se intenta fomentar que el alumno tenga una parte de aprendizaje autónomo, así como el acercamiento a la realidad laboral, con el objeto de que el alumno pueda desempeñar su futura actividad profesional de manera exitosa. Teniendo en cuenta que el mercado laboral está transformándose constantemente y a una velocidad cada vez mayor, es fundamental que los estudiantes adquieran, en sus procesos de aprendizaje, competencias que les proporcionen esa capacidad de adaptación permanente al cambio. Conseguir que los alumnos, cada vez más acostumbrados a los medios digitales e interactivos, estén motivados durante las clases tanto teóricas como prácticas supone un reto para los docentes universitarios. Los estudiantes de hoy representan la primera generación en crecer

\footnotetext{
Iván García Miranda*

ivan.garcia.miranda@uab.cat

Alfonso Durán Heras **

duran@ing.uc3m.es

* Parc de Recerca Universidad Autónoma Barcelona. Avinguda de Can Donènech s/n. 08193 Bellaterra (Barcelona).

** Área de Ingeniería de Organización. Dpto. de Ingeniería

Mecánica. Universidad Carlos III de Madrid. Avda. de la

Universidad, 30. 28911 Leganés (Madrid).
}

con la nueva tecnología digital. Desde la infancia, han experimentado con computadoras, videojuegos, reproductores de música digital, cámaras de video, teléfonos móviles, y todas las demás herramientas de la era digital. Estos 'nativos digitales' tienen una capacidad de atención limitada, menor tolerancia para la repetición, aburrimiento de medios estáticos, y un estilo de aprendizaje más visual que sus antecesores (García et al., 2007).

Otro de los retos a los que los docentes se enfrentan en la actualidad es la falta de interacción entre profesor y alumno en las clases magistrales, llevando al alumno a una falta de interés y de concentración que implica un menor aprovechamiento de la clase. La interacción estudiante profesor ha sido objeto de interés desde hace varios años, siendo estudiada por Edmund Saas (Saas, 1989) o Lee Morganett (Morganett, 1995) entre otros.

Los juegos de ordenador son considerados como un medio de motivación para los alumnos que puedan carecer de interés o confianza (Klawe, 1994) siendo una herramienta valiosa para mejorar el aprendizaje. Se dice que pueden ser particularmente eficaces cuando están diseñados para abordar un problema específico o enseñar una cierta habilidad (Griffiths, 2002), por ejemplo, en el fomento del aprendizaje en materias como matemáticas, física y lenguaje, donde los objetivos específicos pueden especificarse (Randel et al., 1992).

Con estos antecedentes se planteó el proyecto de innovación docente en el área de Ingeniería de Organización para introducir el uso de otro tipo de metodologías en las clases. Los objetivos de esta nueva implantación en una asignatura del Área fueron: 
1. Identificar e implantar una nueva herramienta docente que aumentara la dinámica de la asignatura.

2. Aumentar la motivación de los alumnos.

3. Mejorar la comprensión de conceptos abstractos impartidos en la asignatura.

\section{Teoría y antecedentes}

La utilización de metodologías activas basadas en las nuevas tecnologías es un elemento clave en la actualización de los procesos de enseñanza y aprendizaje en la universidad (Salinas, 2004). James Paul Gee (2003) sostiene que los videojuegos incorporan todo un conjunto de principios de aprendizaje que pueden usarse en otros entornos, por ejemplo, en la enseñanza de la ciencia. Se pueden usar videojuegos y tecnologías de juego para mejorar el aprendizaje ya que tienen niveles iniciales que se han diseñado específicamente para proporcionar a los usuarios los conocimientos básicos necesarios para que puedan construir generalizaciones que les permitan hacer frente a problemas más complejos. Los juegos de simulación se pueden utilizar para presentar temas de ingeniería con otros enfoques que no son posibles dentro de las limitaciones de la clase tradicional. El uso de animaciones, gráficos y un entorno interactivo pueden ser útiles para estimular a los estudiantes y para explicar e ilustrar distintos conceptos, así como para mejorar las habilidades de resolución de problemas (Philpot et al., 2003). La aplicación de juegos serios surge como un método de aprendizaje prometedor en la educación superior (Pourabdollahian et al., 2012).

Otro de los beneficios de la introducción de videojuegos en la enseñanza superior es conseguir aumentar la motivación y la retención (Decker et al., 2013). La parte interactiva es altamente valorada por los alumnos y la participación en clase aumenta (Iosup et al., 2014). El grado de interés de los alumnos aumenta al introducir juegos en la asignatura (Chwif et al., 2003). Además, las estrategias de enseñanza basadas en el juego pueden contribuir al desarrollo de competencias, tanto específicas como transversales, al mismo tiempo que pueden aumentar la motivación de los estudiantes por el aprendizaje (González et al., 2015). Este grado de motivación puede ser más baja en los estudiantes de ingeniería donde el desgaste puede causar el abandono de la carrera (Baillie et al., 2000). Los estudiantes evaluados en este estudio presentado encuentran que los conceptos teóricos y matemáticos son demasiado altos.

Los juegos exploratorios e interactivos son buenos vehículos para introducir conceptos de matemáticas y ciencias que pueden ser difíciles de visualizar o manipular con materiales concretos (Ke, 2008) (Baker et al., 2008). Adivinanzas y videojuegos interactivos han sido utilizados con éxito con estudiantes universitarios para mejorar la creatividad y otras formas de pensamiento crítico (Doolittle 1995). Vogel et al. (2006) profundiza en los resultados de mejoras cognitivas utilizando juegos y simulaciones interactivas en determinadas situaciones. Uno de los beneficios reportados de los juegos de simulación en la enseñanza de la ingeniería industrial fue una mejor comprensión de la dinámica a nivel empresarial (Cox et al., 2004 y Léger, 2006). El estudio de Deshpande et al. (2011) recoge las experiencias previas en el uso de videojuegos en las distintas ingenierías, comprobando que para el área de Ingeniería de Organización los ámbitos donde más se habían aplicado eran: ERPs, Gestión de cadena de suministros y Organización y planificación de la Producción. Los resultados confirmaron que los juegos de simulación ayudaron a los estudiantes a aumentar su conciencia de los problemas del mundo real y comprensión de asignaturas del curso.

Más específicamente, se pudo observar que el uso de "juegos serios" se puede considerar como una herramienta para generar en los alumnos una mentalidad emprendedora (Bellotti et al., 2014). La mentalidad emprendedora supone una de las competencias generales a desarrollar en los estudiantes de Ingeniería, y específicamente en la asignatura de Fundamentos de Gestión Empresarial. La recopilación de beneficios señalados en la aplicación de videojuegos en la enseñanza superior y el potencial de la gamificación mostrado en el artículo de Arnold (2014) impulsaron el desarrollo de un proyecto para incluir el uso de un videojuego serio en un grupo (como prueba) de la asignatura de Fundamentos de Gestión Empresarial, del área de Ingeniería de Organización en el curso 2014/2015 en la universidad Carlos III de Madrid. Es una asignatura troncal de 6 créditos de $2^{\circ}$ curso en todos los grados de Ingeniería. El objetivo de esta asignatura es proporcionar a los futuros ingenieros un conocimiento adecuado del concepto de empresa, su marco institucional, jurídico y medioambiental, así como los fundamentos de la organización y gestión de las empresas. Adicionalmente, pretende transmitir la importancia del papel de la ingeniería y del ingeniero en la gestión empresarial. Las competencias específicas de la asignatura que los alumnos adquieren son:

- Conocimiento adecuado del concepto de empresa, marco institucional y jurídico de la empresa. Organización y gestión de empresas.

- Conocimientos y capacidades adecuados para organizar y dirigir empresas.

Para algunos alumnos de Ingeniería, comprender el concepto de empresa y entender el papel de la misma dentro de la sociedad y las interrelaciones entre los diferentes subsistemas es algo complejo si no se han relacionado con el mundo empresarial. Uno de los elementos que pueden ser de utilidad para afianzar los contenidos teóricos es realizar visitas a empresas de la zona, de manera coordinada con las clases teóricas, con lo que se consigue que los alumnos vean la aplicación práctica de lo visto en clase a empresas de su entorno, estudiando a la vez la adaptación de los sistemas 
teóricos a las peculiaridades de cada empresa, tal y como describió Josep Capó-Vicedo (Capó-Vicedo, 2010). Sin embargo, no siempre es posible realizar visitas a las empresas con grupos numerosos por lo que se procedió a experimentar con un entorno de simulación virtual empresarial.

\section{Metodología}

El proyecto para incluir el uso de un videojuego en la asignatura de Fundamentos de Gestión Empresarial del Grado de Ingeniería Energética comenzó con una revisión de los videojuegos serios para su uso en educación y relacionados con los contenidos de la asignatura. En concreto se hizo una búsqueda de juegos de simulación empresarial. Los juegos de simulación empresarial actúan como juegos serios que contribuyen al aprendizaje a través de una simulación de situaciones de la vida real y entornos empresariales. Son especialmente útiles en el área de procesos de gestión empresarial y estrategias empresariales (Blažič et al., 2012). La combinación de tecnología de simulación y los contenidos teórico-prácticos de la asignatura ofrece a los alumnos una oportunidad sin riesgos para probar una serie de estrategias relevantes para ver la aplicación de los conocimientos adquiridos y ver las consecuencias en los resultados empresariales de un determinado negocio. Los criterios establecidos para la búsqueda de los juegos de simulación fueron:

1. Coste. Siendo prioritario que el coste fuera bajo o incluso gratis su uso (modo prueba durante un tiempo determinado).

2. Requerimientos técnicos. La búsqueda se priorizó para que el acceso al juego fuera mediante una plataforma web para que cualquier alumno pudiera acceder al mismo.

3. Propiedades de usabilidad. En este criterio se engloban distintos aspectos: si el juego está diseñado para un área concreta de la empresa o simula el comportamiento de la empresa completa. Si el juego es competitivo o no competitivo. Si está enfocado en decisiones a corto o a largo plazo. El nivel de preparación necesario para empezar a jugar, etc.
Con el fin de seleccionar los juegos de negocios para la evaluación, se realizó una búsqueda en la red, y donde se encontraron innumerables juegos computarizados que se describen como juegos de negocios. Por ejemplo, en Wikipedia, existe una lista de juegos de negocios con cientos de juegos que están listados en orden alfabético y cronológico. Con la lista de juegos se aplicaron los criterios descritos anteriormente y se obtuvieron dos juegos que encajaban con los requisitos: VIRTONOMICS y HOT SHOT BUSINESS.

Entre los dos preseleccionados, se eligió VIRTONOMICS (https://virtonomics.com), ya que cuenta con una comunidad muy amplia de usuarios y con foros para resolver dudas e incidencias. Además, incluye un registro donde el jugador (en nuestro caso también alumno) introduce sus datos y la actividad y los resultados son trazables, lo que permite una posterior evaluación de la simulación realizada. Virtonomics es un juego multijugador masivo en línea (MMOG - massively multiplayer online game). El objetivo principal del jugador es crear y administrar una empresa con diferentes subdivisiones (como tiendas, fábricas, centros de salud, etc.). El jugador debe controlar el comercio, la producción, la investigación científica, la minería, la agricultura, la administración de personal, las finanzas, el marketing, la logística y otros procesos comerciales. El juego proporciona "feedback" sobre la calidad de la administración y la eficiencia de la empresa. Esta información se proporciona en diferentes hojas, y sin consejos específicos sobre estrategias o acciones que se deben tomar para resolver problemas. En la actualidad el juego dispone de tres versiones (Entrepreneur, Business Wares y Tycoon) aunque en el momento de palicación del proyecto solamente había disponible una de ellas, equivalente a la versión actual de Tycoon.

Las decisiones más destacadas en la simulación empresarial ayudan a comprender mejor una serie de conceptos que se desarrollan en el programa de la asignatura. En la tabla 1 se reflejan las principales decisiones que el usuario puede tomar y el contenido del programa de la asignatura de Fundamentos de Gestión Empresarial al que se hace referencia: 
Tabla 1 Principales decisiones en el simulador empresarial y los conceptos del programa de la asignatura.

\begin{tabular}{|c|c|}
\hline Decisiones a realizar en el simulador & Conceptos del programa de la asignatura \\
\hline $\begin{array}{l}\text { Decidir el sector y la tipología de negocio a la que se va a dedicar } \\
\text { la empresa. }\end{array}$ & La empresa. Tipos y objetivos. \\
\hline $\begin{array}{l}\text { Decidir la cantidad de recursos financieros que se emplean en } \\
\text { producción y los que se emplean en ventas. }\end{array}$ & $\begin{array}{l}\text { Asignación de recursos y división de trabajo. Funciones y pro- } \\
\text { cesos empresariales. }\end{array}$ \\
\hline $\begin{array}{l}\text { Decidir la cantidad de materias primas que se compra y gestión } \\
\text { de stocks de materia prima. }\end{array}$ & Logística y gestión de la cadena de suministros. \\
\hline $\begin{array}{l}\text { Decidir si se implanta un aprovisionamiento automático con el } \\
\text { proveedor. }\end{array}$ & Logística y gestión de la cadena de suministros. \\
\hline Elegir si un producto lo compro o lo fabrico. & La decisión de inversión. Sistemas productivos. \\
\hline $\begin{array}{l}\text { Identificar y decidir el portfolio de productos que se ponen a } \\
\text { la venta. }\end{array}$ & Gestión comercial y marketing. \\
\hline Establecer los precios de venta de los bienes. & Gestión comercial y marketing. \\
\hline $\begin{array}{l}\text { Decidir la cantidad de recursos humanos que se asignan a cada } \\
\text { función (producción, venta, I+D). }\end{array}$ & La gestión de los recursos humanos. La función directiva. \\
\hline Decidir en qué productos se llevan a cabo tareas de branding. & Gestión comercial y marketing. \\
\hline $\begin{array}{l}\text { Decidir la cantidad de recursos financieros para campañas de } \\
\text { publicidad. }\end{array}$ & Gestión comercial y marketing. \\
\hline Decidir el plan de medios para la campaña de publicidad. & El plan de marketing. \\
\hline $\begin{array}{l}\text { Decidir en qué país se realiza la producción/compra de produc- } \\
\text { tos. }\end{array}$ & La dirección de la empresa. Funciones y procesos empresariales. \\
\hline Asignación de recursos en determinadas fábricas. & Gestión de producción. Impuestos y aduanas. \\
\hline Decidir los recursos que se asignan a la mejora de la tecnología. & La dirección de la empresa. Funciones y procesos empresariales. \\
\hline
\end{tabular}

Tras el estudio de las opciones del juego, simulaciones y pruebas de funcionalidad, se establecieron unas instrucciones para los alumnos con el fin de establecer un marco común para poder realizar una evaluación de los resultados obtenidos. Según los contenidos y el avance de la asignatura, se decidió que la simulación comenzara el 25 de Marzo 2015, donde los alumnos ya habían visto más del $50 \%$ del contenido de la asignatura y fueran utilizando el juego de simulación empresarial hasta el 6 de Mayo 2015. En las instrucciones para los alumnos se establecieron los requisitos para que el trabajo realizado fuese valorable, dichos requisitos fueron:

1. Que el usuario creado en el juego fuera el número de identificación del alumno (NIA).

2. Que el informe solicitado con los estados financieros de la compañía creada estuviera subido antes de las 23.59h. del día 7 de Mayo.

Cabe destacar el hecho de que se impuso una competición individual, de forma que los alumnos que mejores resultados empresariales obtuvieran, tendrían las calificaciones más altas. De un total de 46 alumnos que había en el grupo, los 5 mejores obtendrían 0,5 puntos, los 5 siguientes obtendrían 0,4, los 5 siguientes obtendrían 0,3 y el resto, obtendrían
0,2 puntos. No obstante, para recibir la calificación de 0,2 puntos había que tomar decisiones empresariales durante un mínimo de una semana (cada turno dura un día, lo que implica que las decisiones realizadas en un turno no se ven reflejadas hasta el día siguiente). Se realizó la presentación a los alumnos con un esquema de actividad voluntaria por parte de los alumnos y con una pequeña bonificación sobre la nota de evaluación continua para aquellos alumnos que la realizaran (máximo de 0,5 puntos adicionales), siendo dicha bonificación menor del esfuerzo necesario para llevar a cabo la actividad.

\section{Resultados}

El número de estudiantes que decidieron realizar la actividad con el juego fue de 17 alumnos, aunque uno de ellos no cumplió con uno de los requisitos exigidos, por lo que 16 alumnos finalizaron con éxito las tareas exigidas en el juego. La duración del juego fue de 4 semanas, en las que los alumnos debían de tomar decisiones sobre su negocio, para, al final, presentar la cuenta de resultados y el balance. Para evaluar la bondad de sus decisiones se realizó una comparativa con el indicador ROE (Return On Equity), un indicador que mide la rentabilidad del capital del accionista y que equivale al cociente entre el beneficio neto y el patrimonio neto. 
Aunque no son datos críticos para el objetivo del proyecto, cabe destacar en lo referente a la actuación de los alumnos sobre el devenir de la simulación empresarial:

Solamente 4 de los 16 alumnos (25\%), no obtuvieron beneficios en ninguna de las cuatro semanas.

Un total de 4 de los 16 alumnos (25\%) obtuvieron un ROE positivo después de las cuatro semanas de simulación.

Sin embargo, el objetivo del proyecto no era analizar el desempeño de los alumnos en la simulación empresarial sino analizar el impacto del uso de una nueva metodología basada en gamificación en una asignatura del área de Ingeniería de Organización, por lo que para ello, se llevó a cabo una encuesta entre los alumnos que realizaron la actividad mediante un formulario de Google incrustado en la plataforma Moodle para el grupo de alumnos de la asignatura. Las preguntas de la encuesta fueron:

P1. El juego de Virtonomics me ayudó a entender mejor los conceptos aprendidos en clase.

P2. El juego de Virtonomics me ayudó a estar más motivado en clase.

P3. El juego de Virtonomics me ayudó a entender mejor el espíritu emprendedor.

Figura 1 Resultados P1: El juego de Virtonomics me ayudó a entender mejor los conceptos aprendidos en clase.
P4. Me gustaría tener más juegos como este en otras asignaturas.

P5. El juego Virtonomics debería incluirse en los próximos cursos de esta asignatura.

Para la valoración de estas cuestiones se utilizó una escala Likert con la siguiente escala de valores:

1. Totalmente de desacuerdo

2. En desacuerdo

3. Neutral

4. De acuerdo

5. Totalmente de acuerdo

Los porcentajes de las respuestas obtenidas en cada una de las preguntas formuladas se muestran gráficamente a continuación:

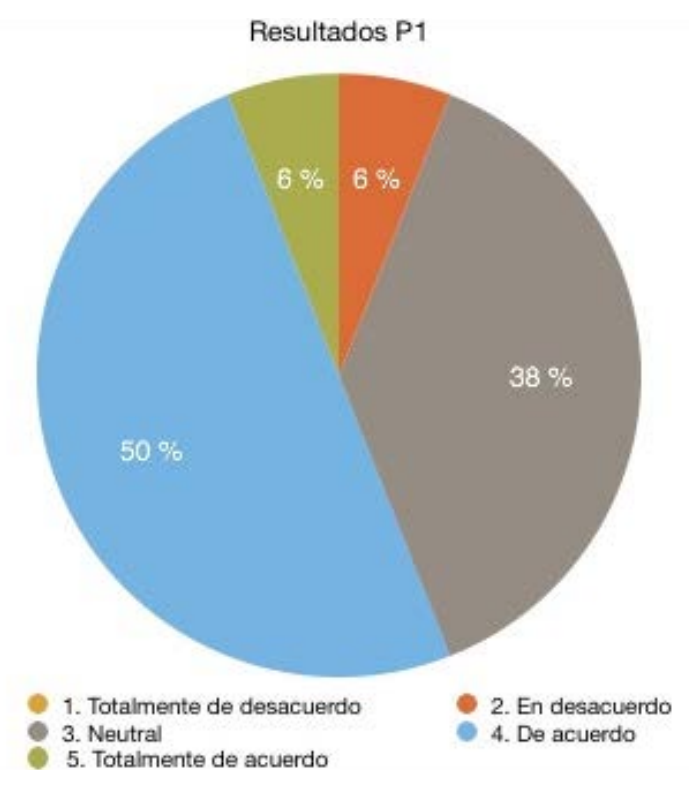

Los resultados muestran que los alumnos mayoritariamente $(>50 \%)$ creen que el uso del juego Virtonomics supuso una ayuda para comprender mejor los conceptos vistos en la asignatura, siendo por tanto, una herramienta de apoyo a la docencia. 
Figura 2 Resultados P2: El juego de Virtonomics me ayudó a estar más motivado en clase.

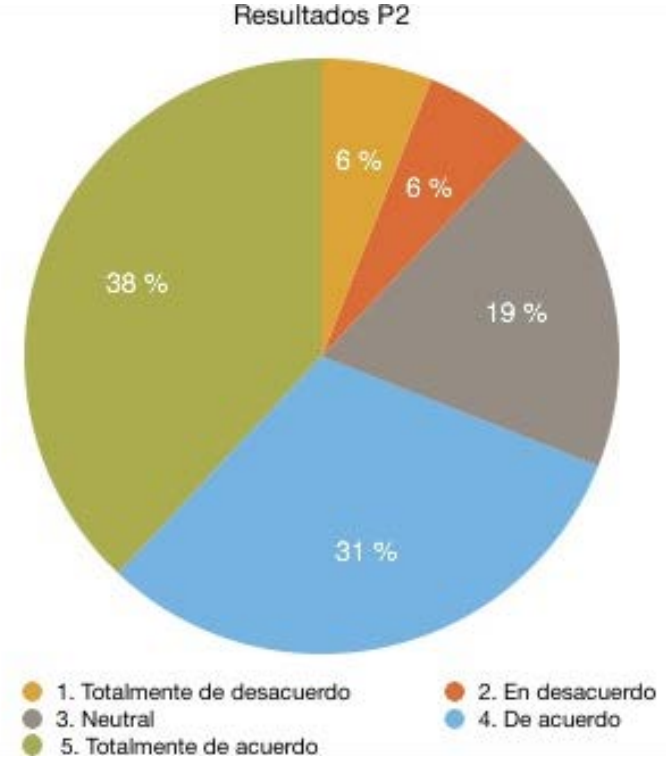

Por ello, se puede afirmar que el uso del juego supuso un aumento en la motivación de los alumnos a la hora de cursar la asignatura de Fundamentos de Gestión Empresarial. de la motivación del alumnado, se observa como un $69 \%$ de las respuestas obtenidas están de acuerdo, siendo un mínimo $12 \%$ el porcentaje de respuestas que están en desacuerdo.

Figura 3 Resultados P3: El juego de Virtonomics me ayudó a entender mejor el espíritu emprendedor.

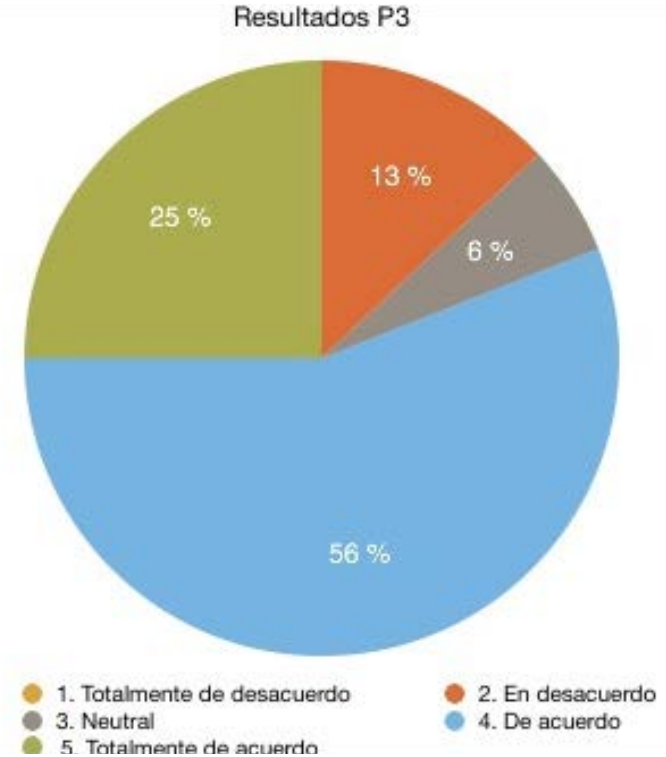

La pregunta P3 supone una evaluación sobre si el juego de simulación empresarial constituyó una herramienta para entender mejor el espíritu emprendedor a los alumnos. Un $81 \%$ de los alumnos están de acuerdo y únicamente un $13 \%$ consideró estar en desacuerdo con la cuestión. Los resultados por tanto, confirman que el uso de juegos de simulación empresarial ayuda a los alumnos de estudios superiores a comprender el espíritu emprendedor. 
Figura 4 Resultados P4: Me gustaría tener más juegos como este en otras asignaturas.

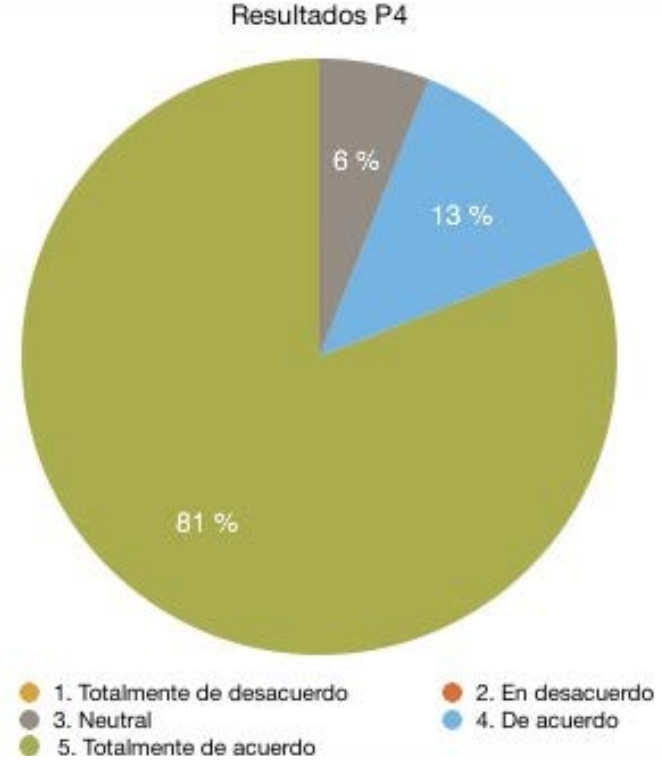

La pregunta P4 hace referencia a si a los alumnos les gustaría tener otros juegos en las distintas asignaturas y los resultados muestran un $94 \%$ de respuestas afirmativas por lo que se puede confirmar que los alumnos están a favor de la introducción de herramientas de gamificación en las asignaturas.
Figura 5 Resultados P5: El juego

Virtonomics debería incluirse en los próximos cursos de esta asignatura.

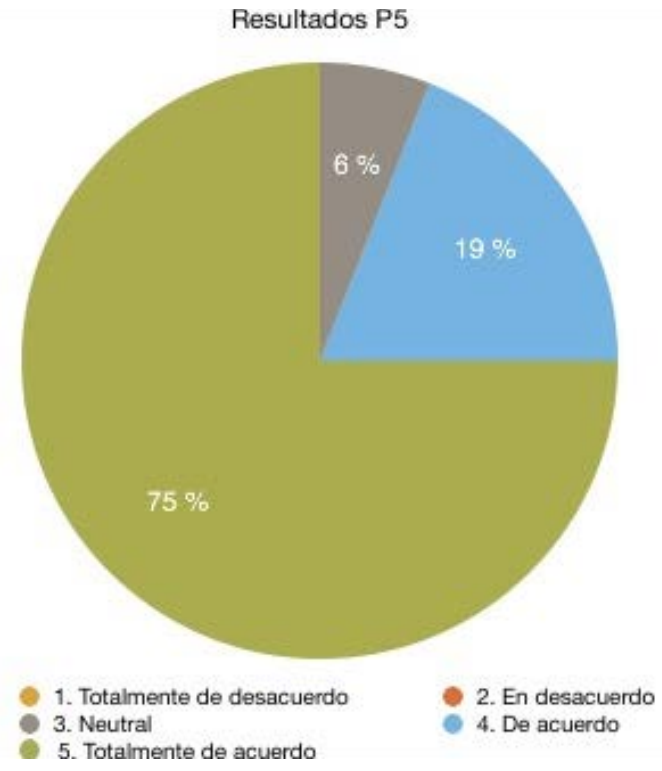

Como pregunta final, se consultó si consideraban que el juego Virtonomics debería de ser incluido en los cursos de la misma asignatura para los próximos cursos, obteniendo una respuesta masivamente positiva (94\%) por lo que se puede confirmar que los alumnos recomendarían el uso de este juego de simulación empresarial en un futuro. 


\section{Conclusiones}

El proyecto descrito en este artículo pretendía cubrir varios objetivos generales en el ámbito de innovación docente:

1. Usar eficazmente otras herramientas TIC interactivas.

2. Utilizar nuevas herramientas docentes.

3. Utilizar un entorno dinámico e interactivo.

Además de otros objetivos orientados a que los alumnos tuvieran un mejor proceso de aprendizaje:

4. Aumentar el interés por la asignatura.

5. Mejorar la motivación de los alumnos para mejorar el sistema enseñanza-aprendizaje.

6. Mejorar la comprensión de conceptos abstractos.

7. Fomentar el emprendimiento de los alumnos.

La realización del proyecto permitió corroborar el uso de videojuegos de simulación empresarial como herramienta interactiva de éxito entre los alumnos. Esta nueva herramienta docente en asignaturas del área de Ingeniería de Organización mejora el entorno del sistema de enseñanza-aprendizaje suponiendo una novedad para los alumnos de estudios superiores y algo que ellos recomendarían su uso posterior en la misma asignatura y en otras asignaturas.

Los resultados obtenidos en una encuesta tras la realización de la prueba de uso del videojuego de simulación confirmaron que la motivación de los alumnos aumenta, implicando un aumento del interés por la asignatura, confirmando los resultados presentados en 2016 por Elhag (2016). Además, se confirmó que los alumnos pudieron comprender mejor los conceptos de la asignatura con el uso del juego descrito, mejorando la adquisición de las competencias específicas de la asignatura. De forma adicional, el juego de simulación supuso una mejora en el espíritu emprendedor de los alumnos que llevaron a cabo la tarea. Sin embargo, todos estos beneficios suponen un coste para el docente (Iosup et al., 2014). Además del tiempo necesario, hay una necesidad de gestión, especialmente para cursos con numerosos alumnos, donde es necesario explicar un nuevo sistema a los estudiantes y vencer la inercia organizativa para obtener la aprobación.

\section{Referencias bibliográficas}

ARNOLD, B. J. (2014). "Gamification in education". Proceedings of the American Society of Business and Behavioral Sciences, 21(1), 32-39.
BAKER, R., WALONOSKI, J., HEFFERNAN, N., ROLL, I., CORBETT, A. \& KOEDINGER, K. (2008). "Why Students Engage in "Gaming the System". Behavior in Interactive Learning Environments”. Journal of Interactive Learning Research, 19(2), 185-224. Chesapeake, VA: AACE.

BAILLIE, C.; FITZGERALD, G. (2000). "Motivation and attrition in engineering students". European Journal of Engineering Education, 2000, vol. 25, no 2, p. 145-155.

BELLOTTI, F., BERTA, R., DE GLORIA, A., LAVAGNINO, E., ANTONACI, A., DAGNINO, F., ... \& MAYER, I. S. (2014). "Serious games and the development of an entrepreneurial mindset in higher education engineering students". Entertainment Computing, 5(4), 357-366.

BLAŽIČ, A. J., RIBEIRO, C., FERNANDES, J., PEREIRA, J., \& ARH, T. (2012). “Analysing the required properties of business simulation games to be used in e-learning and education”. Intelligent Information Management, 4(6), 348-356.

CAPÓ-VICEDO, J. (2010). “Docencia de asignaturas de gestión en una ingeniería. Utilización de metodologías activas de aprendizaje”. Revista de Formación e Innovación Educativa Universitaria, 3(2), 97-111.

CHWIF, L.; BARRETTO, M. R. P. (2003). "Perspectives on simulation in education and training: simulation models as an aid for the teaching and learning process in operations management”. Proceedings of the 35th conference on Winter simulation: driving innovation. Winter Simulation Conference, 2003. p. 1994-2000.

COX, J. F.; WALKER, E. D. (2004), "Using a Socratic Game to Introduce Basic Line Design and Planning and Control Concepts”. Decision Sciences Journal of Innovative Education, 2: 77-82. doi:10.1111/j.00117315.2004.00022.x

DECKER, A.; LAWLEY, E. L. (2013). "Life's a game and the game of life: how making a game out of it can change student behavior". Proceeding of the 44th ACM technical symposium on Computer science education. ACM, 2013. p. 233-238.

DEL FAVERO, L., BOSCOLO, P., VIDOTTO, G., y VICENTINI, M. (2007). "Classroom discussion and individual problem-solving in the teaching of History: Do different instructional approaches affect interest in different ways?". Learning and Instruction, 17, pp. 635657. 
DESHPANDE, A.A.; HUANG, S.H.(2011). “Simulation games in engineering education: A state of the art review”. Computer applications in engineering education, 2011, vol. 19, no 3, p. 399-410.

DOOLITTLE J.H. 1995. "Using riddles and interactive computer games to teach problem-solving skills”. Teaching of Psychology, 22(1), 33-36.

ELHAG, S. M. G. (2016). “Game based Learning and using Course Learning, Collaboration and Management Tools in Black Board”. International Journal of Computer Applications, 154(2), 21-24.

GARCÍA, F.; PORTILLO, J.; ROMO, J.; \& BENITO, M. (2007). "Nativos digitales y modelos de aprendizaje". SPDECE, 2007. https://www.businessintelligence.info/ assets/varios/nativos-digitales.pdf. Visitado 19 marzo 2019.

GEE, J. P. (2003). "What video games have to teach us about learning and literacy". Computers in Entertainment (CIE), 2003, vol. 1, no 1, p. 20-20.

GONZÁLEZ, C. S.; MORA, A. (2015). “Técnicas de gamificación aplicadas en la docencia de Ingeniería Informática”. ReVisión, 2015, vol. 8, no 1.

GRIFFITHS, M.D. (2002). "The educational benefits of videogames". Education and Health, 20(3), pp. 47-51.

IOSUP, A.; EPEMA, D. (2014). “An experience report on using gamification in technical higher education". Proceedings of the 45th ACM technical symposium on Computer science education. ACM, 2014. p. 27-32.

KE, F. (2008). "A case study of computer gaming for math: Engaged learning from gameplay?”. Computers \& Education, 51(4), 1609-1620.

KLAWE, M.M. (1994). "The educational potential of electronic games and the E-GEMS Project”. In T Ottman and I Tomek (eds) Proceedings of the ED-MEDIA 94. World Conference on Educational Multimedia and Hypermedia. Panel discussion 'Can electronic games make a positive contribution to the learning of mathematics and science in the intermediate classroom?' AACE (Association for the Advancement of Computing in Education), Vancouver, Canada, 25-30 June 1994.
LÉGER, P.M. (2006). “Using a simulation game approach to teach ERP concepts”. HEC Montréal, Groupe de recherche en systèmes d'information, 2006.

MORGANETT, L. (1995). “Ten tips for improving teacher-student relationships”. Social Education, 59 (1), pp. 27-28.

PHILPOT, T. A.; HUBING, N.; HALL, R. H.; FLORI, R. E.; OGLESBY, D. B.; YELLAMRAJU, V. (2003). "Games as teaching tools in engineering mechanics courses". In American Society for Engineering Education Annual Conference \& Exposition (pp. 22-25). American Society for Engineering Education ASEE (2003).

POURABDOLLAHIAN, B.; TAISCH, M.; KERGA, E. (2012). "Serious games in manufacturing education: Evaluation of learners' engagement”. Procedia Computer Science, 2012, vol. 15, p. 256-265.

RANDEL J.M., MORRIS B.A., WETZEL C.D., WHITEHILL B.V. 1992. "The effectiveness of games for educational purposes: a review of recent research”. Simulation and Gaming, 23(3), 261-276.

SASS, E. J. (1989). "Motivation in the College Classroom: What Students Tell Us”. Teaching of Psychology.16 (2), pp. 86-88.

SALINAS, J. (2004). “Innovación docente y uso de las TIC en la enseñanza universitaria”. International Journal of Educational Technology in Higher Education (ETHE), 1(1).

VOGEL, J. J., VOGEL, D. S., CANNON-BOWERS, J., BOWERS, C. A., MUSE, K., \& WRIGHT, M. (2006). Computer gaming and interactive simulations for learning: A meta-analysis. Journal of Educational Computing Research, 34(3), 229-243.

WOOTEN, T.C. (1998). "Factors influencing student learning in introductory Accounting Classes: a comparison of traditional and non-traditional students". Issues in Accounting Education, 13(2), pp. 357-373. 